(C) 1998 International Press

Adv. Theor. Math. Phys. 2 (1998) 61-90

\title{
New "Gauge" Theories in Six Dimensions
}

\author{
Edward Witten ${ }^{1}$ \\ School of Natural Sciences, Institute for Advanced Study \\ Olden Lane \\ Princeton, NJ 08540, USA
}

\begin{abstract}
More general constructions are given of six-dimensional theories that look at low energy like six-dimensional super Yang-Mills theory. The constructions start with either parallel fivebranes in Type IIB, or $M$-theory on $\left(\mathbb{C}^{2} \times \mathbb{S}^{1}\right) / \Gamma$ for $\Gamma$ a suitable finite group. Via these constructions, one can obtain six-dimensional theories with any simple gauge group, and $S U(r)$ theories with any rational theta angle. A matrix construction of these theories is also possible.
\end{abstract}

\section{Introduction}

One of the fascinating results obtained in investigations of string duality and matrix theory is that there exist in six dimensions theories that do not have dynamical gravity, but have many properties of string theory, such as $T$-duality upon toroidal compactification. Such theories can be obtained by taking the weak coupling limit of a system of parallel Type II or heterotic string fivebranes [1].

In some cases, these theories have a quite interesting and exotic infrared behavior, reducing for example at long distances to the exotic sixdimensional theory that can be constructed from Type IIB at an ALE singularity [2] or via parallel $M$-theory fivebranes [3]. Other examples look at long distances like infrared-free super-Yang-Mills theories. The focus of the present paper will be on such "gauge" theories. We will furthermore

\footnotetext{
${ }^{1}$ Research Supported in part by NSF Grant PHY-9513835
} 
consider only examples that have the maximal possible supersymmetry for six-dimensional gauge theories, namely $(1,1)$ supersymmetry. (There are also many examples of such theories with $(0,1)$ supersymmetry, as explored recently in [4].)

Sections 2-5 are devoted to a class of examples that can be constructed from a Type IIB system with parallel NS and D fivebranes, or alternatively via an $M$-theory construction that we present. These theories have unitary gauge groups at low energies, but differ from previously studied systems with such gauge groups; in fact, we compute a specific difference (involving a spacetime theta angle) in section 5. Section 6 is devoted to examples with orthogonal and symplectic gauge groups, which can be constructed from Type IIB or from $M$-theory, and examples with exceptional gauge groups, including $G_{2}$ and $F_{4}$, for which we have only an $M$-theory construction. A recent analysis [5] of $S$-duality in $\mathcal{N}=4$ super Yang-Mills with non-simplylaced gauge groups is closely related to and could be formulated in terms of the six-dimensional theories studied in section 6. And finally, in section 7 we reexamine the $S U(r)$ theories of sections 2-5 from the standpoint of matrix theory, interpreting them via the Coulomb branch of a certain twodimensional $(4,4)$ super Yang-Mills theory. Issues related to this disccussion have also been discussed recently in [6].

\section{$2(p, q)$ Fivebrane System}

On the world-volume of a collection of $q$ identical parallel fivebranes in Type IIB superstring theory, one sees at low energies a six-dimensional super YangMills theory with gauge group $U(q)$. This is so for either Dirichlet or NS fivebranes. In the case of NS fivebranes, the gauge coupling in this $U(q)$ gauge theory is independent of the string coupling constant $\lambda$. As a result of this [1], in the limit that $\lambda$ goes to zero, although the Type IIB string theory becomes free in bulk, the theory on the fivebranes does not become free. To be more precise, it seems very likely (and is implied by a proposed $T$-duality [7] that will be reviewed and generalized in section 4) that the center of $U(q)$ decouples as $\lambda \rightarrow 0$, and that in this limit there is a surviving six-dimensional supersymmetric gauge theory with $S U(q)$ gauge group. This theory has six-dimensional $(1,1)$ supersymmetry and is a non-chiral theory with 16 supercharges, in common with all other six-dimensional theories that will be discussed in the present paper.

It is natural to combine $\lambda$ with the Type IIB theta angle $\theta_{B}$ to a parameter $\tau=\theta_{B} / 2 \pi+i / \lambda$ with values in the upper half plane. The Type IIB superstring theory is believed to have an $S L(2, \mathbb{Z})$ symmetry that acts by

$$
\tau \rightarrow \frac{a \tau+b}{c \tau+d}
$$


with $a, b, c, d \in \mathbb{Z}$ and $a d-b c=1$. The limit $\lambda \rightarrow 0$ corresponds to $\tau \rightarrow i \infty$. One might ask whether the limiting theory, obtained by taking $\tau \rightarrow i \infty$, depends on $\theta_{B}$. We will give some arguments that it does not.

In this paper, we will study the more general case of a collection of $q$ NS fivebranes and $p$ Dirichlet fivebranes. If one only wishes to determine the low energy gauge group, which is expected to be independent of $\tau$, one can apply an $S L(2, \mathbb{Z})$ transformation, ignore the action of this transformation on $\tau$, and map the $(p, q)$ fivebrane system (the two integers will always be respectively the Dirichlet and NS fivebrane charges) to an $(r, 0)$ system, where $r$ is the greatest common divisor of $p$ and $q$. The low energy gauge group is hence $U(r)$.

If, however, we wish to extract a decoupled six-dimensional theory by taking $\tau \rightarrow i \infty$, then we should restrict ourselves to $S L(2, \mathbb{Z})$ transformations which commute with this limit. This means that we should limit ourselves to upper triangular matrices

$$
\left(\begin{array}{ll}
a & b \\
c & d
\end{array}\right)=\left(\begin{array}{ll}
1 & n \\
0 & 1
\end{array}\right)
$$

which act by $\tau \rightarrow \tau+n$. Since the action of $S L(2, \mathbb{Z})$ on the fivebrane charges is

$$
\left(\begin{array}{l}
p \\
q
\end{array}\right) \rightarrow\left(\begin{array}{ll}
a & b \\
c & d
\end{array}\right)\left(\begin{array}{l}
p \\
q
\end{array}\right)
$$

such transformations map $(p, q) \rightarrow(p+n q, q)$. Hence $p$ can be uniquely mapped to the region $0 \leq p \leq q-1$, and for each $q, p$ has precisely $q$ possible values.

The theory obtained by taking $\tau \rightarrow i \infty$ for given $(p, q)$ has low energy gauge group $U(r)$ ( $r$ being again the greatest common divisor of $p$ and $q$ ). For $r>1$, this group is non-abelian. Moreover, the gauge coupling constant of this theory is independent of $\tau$ for $\tau \rightarrow i \infty$ by the same sort of arguments as for $p=0$. Hence, just as in [1], the theory obtained in this way cannot be free if $r>1$. Arguments given below actually imply that it is not free whenever $q>1$. This is the strongest possible statement of its sort since for $q=1$, the generalization to include Dirichlet fivebranes is vacuous, as the transformation $(p, q) \rightarrow(p+n q, q)$ can be used to set $p$ to zero.

It is very plausible (and follows from the duality we will propose) that the $U(1)$ factor in $U(r)$ actually decouples, and that the low energy theory is an $S U(r)$ theory. If so, there is no low energy gauge group at all in the six-dimensional theory when $r=1$. The $M$-theory interpretation to which we turn next nevertheless implies, for $q>1$, that non-trivial six-dimensional physics survives as $\lambda \rightarrow 0$ even when $r=1$. 


\section{$3 \quad M$-Theory Dual}

The goal in this section is to propose, and to begin to explore, an $M$-theory dual of the $(p, q)$ theory. For $p=0$, such a dual has been proposed some time ago [7] and studied from various points of view [8-10]. One considers Type IIA superstring theory at an $A_{q-1}$ singularity. Type IIA perturbation theory breaks down in the field of such a singularity, if all world-sheet theta angles vanish. A non-perturbative $S U(q)$ gauge group appears, with a gauge coupling constant that does not vanish in the limit that the Type IIA coupling constant $\lambda_{A}$ vanishes. Upon taking this limit, one gets a surviving six-dimensional theory with $S U(q)$ gauge symmetry and $(1,1)$ supersymmetry. This theory is believed to be equivalent to the one obtained in the weak coupling limit from $q$ parallel Type IIB fivebranes. We will review in section 4 a version of the $T$-duality argument for this equivalence.

We recall that the $A_{q-1}$ singularity can be described as the singularity at the origin in the quotient space $\mathbb{C}^{2} / \mathbb{Z}_{q} .{ }^{2}$ Here $\mathbb{C}^{2}$ has complex coordinates $z_{1}, z_{2}$, and $\mathbb{Z}_{q}$ acts by

$$
\begin{aligned}
& z_{1} \rightarrow e^{2 \pi i / q} z_{1} \\
& z_{2} \rightarrow e^{-2 \pi i / q} z_{2} .
\end{aligned}
$$

To generalize this dual description of the $(0, q)$ theory to the $(p, q)$ case, we will have to go to $M$-theory. Type IIA on $\mathbb{R}^{6} \times \mathbb{C}^{2} / \mathbb{Z}_{q}$ is the same as $M$-theory on $\mathbb{R}^{6} \times \mathbb{C}^{2} / \mathbb{Z}_{q} \times \mathbb{S}^{1}$. The weak coupling limit of Type IIA is the limit that the radius $R$ of the $\mathbb{S}^{1}$ is small; we let $t, 0 \leq t \leq 2 \pi R$, be an angular variable on $\mathbb{S}^{1}$.

Before going on, let us note a subtlety that will have interesting analogs later. Super Yang-Mills theory in six dimensions with $S U(q)$ gauge group has a moduli space of vacua parametrized by the expectation values of four scalars $\phi^{i}, i=1, \ldots, 4$ in the adjoint representation of $S U(q)$. For the vacuum energy to vanish, they must mutually commute and so can be simultaneously diagonalized. The $k^{\text {th }}$ eigenvalue $\phi_{(k)}^{i}$ of the $\phi^{i}$, for $k=1, \ldots, q$, defines a point in $\mathbb{R}^{4}$. As the $\phi^{i}$ are traceless, $\sum_{k} \phi_{(k)}^{i}=0$. The $\phi^{i}$ thus define $q-1$ independent points in $\mathbb{R}^{4}$. The moduli space $\mathcal{M}$ of vacua is obtained by dividing by the Weyl group, which is the group $\mathbb{S}_{q}$ of permutations of the eigenvalues, so one has

$$
\mathcal{M}=\left(\mathbb{R}^{4}\right)^{q-1} / \mathbb{S}_{q} .
$$

Now, $M$-theory on $\mathbb{C}^{2} / \mathbb{Z}_{q} \times \mathbb{S}^{1}$ has a moduli space which is instead

$$
\widetilde{\mathcal{M}}=\left(\mathbb{R}^{3} \times \mathbb{S}^{1}\right)^{q-1} / \mathbb{S}_{q}
$$

\footnotetext{
${ }^{2}$ To fully exhibit the symmetries, $\mathbb{C}^{2}$ should here really be regarded as a flat hyperKahler manifold $\mathbb{R}^{4}$, without a chosen complex structure.
} 
It differs from $\mathcal{M}$ because one of the four scalars in the six-dimensional effective super Yang-Mills theory originates from the Wilson line around the $\mathbb{S}^{1}$ factor in $\mathbb{C}^{2} / \mathbb{Z}_{q} \times \mathbb{S}^{1}$. This Wilson line is group-valued, not Lie algebra valued, so its eigenvalues live in $\mathbb{S}^{1}$, not in $\mathbb{R}$; taking account of the Wilson line together with three seven-dimensional scalars in the adjoint representation gives (3.3).

The circle in (3.3), because it originates as a holonomy, is the dual of the original circle in $\mathbb{C}^{2} / \mathbb{Z}_{q} \times \mathbb{S}^{1}$. So as we take the radius of the original circle to zero, the radius of the circle that enters in the definition of $\widetilde{\mathcal{M}}$ goes to infinity. Hence, in the decoupled six-dimensional theory, this circle is decompactified. To obtain a six-dimensional theory, one takes the coupling to zero in a vacuum given by some point $P \in \widetilde{\mathcal{M}}$, and in the limit of zero coupling, one only sees a small neighborhood of $P$ (that is, a neighborhood vanishingly small compared to the radius of the $\left.\mathbb{S}^{1}\right)$.

The limiting theory that one gets depends on the choice of $P$. If $P$ is a generic, smooth point in $\widetilde{\mathcal{M}}$, the limiting theory is a free abelian theory; if $P$ is the singularity at which all $q$ points in $\mathbb{R}^{3} \times U(1)$ coincide, the limiting theory is an $S U(q)$ theory; if $P$ is a singular point at which $q^{\prime}$ points coincide $\left(1<q^{\prime}<q\right)$ one gets a theory of the same structure with $S U\left(q^{\prime}\right)$ gauge group. The appearance here of the $S U\left(q^{\prime}\right)$ theory does not have much novelty because it can be obtained in any case by first constructing the sixdimensional $S U(q)$ theory and then Higgsing it. In that sense then, in the present example everything interesting comes by considering the case that $P$ is the maximally singular point. If, however, $\widetilde{\mathcal{M}}$ had more than one maximally singular point, then essentially different six-dimensional theories (no one of which could be obtained by Higgsing another) could arise from small radius limits of $M$-theory on $\mathbb{C}^{2} / \mathbb{Z}_{q} \times \mathbb{S}^{1}$ for different $P$. We will find such situations later in this paper.

\subsection{Generalization}

We propose now to generalize Type IIA at an $A_{q-1}$ singularity to $M$-theory on $\mathbb{R}^{6} \times\left(\mathbb{C}^{2} \times \mathbb{S}^{1}\right) / \mathbb{Z}_{q}$, where $\mathbb{Z}_{q}$ acts by

$$
\begin{aligned}
z_{1} & \rightarrow e^{2 \pi i / q} z_{1} \\
z_{2} & \rightarrow e^{-2 \pi i / q} z_{2} \\
t & \rightarrow t-2 \pi R \frac{p}{q},
\end{aligned}
$$

with integer $p$. We write $X_{p, q}$ for $\left(\mathbb{C}^{2} \times \mathbb{S}^{1}\right) / \mathbb{Z}_{q}$ with this action of $\mathbb{Z}_{q}$. Notice that $p$ is only well-defined modulo $q$, and that in particular if $q=1, X_{p, q}$ is just the original $\mathbb{C}^{2} \times \mathbb{S}^{1}$. So just as in the previous section, the interesting cases are $q \geq 2,0 \leq p<q$. 
We want to consider $M$-theory on $\mathbb{R}^{6} \times X_{p, q}$. In the limit $R \rightarrow 0$, this becomes if one is not very near $z_{1}=z_{2}=0$ equivalent to weakly coupled Type IIA on $\mathbb{C}^{2} / \mathbb{Z}_{q}$. In fact, an observer at a point $P \in \mathbb{C}^{2}$ that is not close to $z_{1}=z_{2}=0$ does not detect by local measurements that $P$ has been identified with $q-1$ image points, and so in particular does not detect the details of how this identification was made. So in bulk, $M$-theory on $\mathbb{R}^{6} \times X_{p, q}$ becomes a free Type IIA theory for $r \rightarrow 0$.

As long as $p$ is not congruent to $0 \bmod q$, the theory near $z_{1}=z_{2}=0$ cannot be a free Type IIA theory, for the following reason. As in section 2, let $r$ be the greatest common divisor of $p$ and $q$, and let $s=q / r$. Thus, $s>1$ if $p$ is not congruent to $0 \bmod q$. As was noted in [11] in a context closely related to the present discussion, the present theory has the following unusual property from a Type IIA point of view. In addition to conventional Type IIA superstrings which can be found at any values of $z_{1}, z_{2}$ and come from $M$-theory membranes that wrap once around $\mathbb{S}^{1}$, the theory has additional strings whose tension is $1 / s$ times the standard Type IIA tension and which propagate only on $\mathbb{R}^{6}$, that is only at $z_{1}=z_{2}=0$. The reason for this is that at $z_{1}=z_{2}=0, \mathbb{Z}_{q}$ is acting only on $\mathbb{S}^{1}$, creating a circle that is $s$ times smaller than the original $\mathbb{S}^{1}$. So near the origin, there are strings that come from membranes that wrap once around $\mathbb{S}^{1} / \mathbb{Z}_{s}$. Such strings are prevented from moving away from a small neighborhood of $z_{1}=z_{2}=0$ by a strong energetic barrier. Their tension is $s$ times smaller than the usual Type IIA string tension because they come from membranes that are $s$ times shorter. (Like the ordinary strings, the exotic ones are BPS saturated, but with a charge $s$ times smaller; so the ratio of tensions is precisely $s$.) $s$ of these strings can combine to an object that can move without energetic cost to large $z_{1}, z_{2}$ and turn into an ordinary Type IIA superstring near infinity.

The theory of weakly coupled Type IIA strings cannot be enriched by adding strings of fractional charge while preserving the existence of a systematic string perturbation expansion. So the presence of these fractional strings shows that the theory with $p$ not congruent to $0 \bmod q$ cannot reduce to a free theory in the limit of small $R$. It is a free Type IIA theory in bulk, but there are surviving interactions at $z_{1}=z_{2}=0$. (Of course, this is also true for $p=0, q>1$ because of the $S U(q)$ gauge symmetry. This is the more familiar case that we discussed first.) In this way, we get a family of six-dimensional theories, with $(1,1)$ supersymmetry, parametrized by the choice of integers $(p, q)$ with $q \geq 2$ and $p \cong p+q$.

What is the low energy gauge group of this theory? It is expected to be independent of $R$ (unless there is a very unfamiliar kind of phase transition) and is conveniently determined by going to large $R$ and using the longwavelength approximation to $M$-theory. Non-abelian gauge symmetry in $M$-theory comes from singularities. In the present case, singularities of $X_{p, q}$ 
come only from fixed points of the $\mathbb{Z}_{q}$ action on $\mathbb{C}^{2} \times \mathbb{S}^{1}$. Since $\mathbb{Z}_{q}$ acts on $\mathbb{S}^{1}$ by translations, any element of $\mathbb{Z}_{q}$ that acts non-trivially on $\mathbb{S}^{1}$ acts freely on $\mathbb{S}^{1}$ and hence on $\mathbb{C}^{2} \times \mathbb{S}^{1}$. Fixed points and singularities come therefore only from the subgroup of $\mathbb{Z}_{q}$ that acts trivially on $\mathbb{S}^{1}$. This subgroup is isomorphic to $\mathbb{Z}_{r}$. Its action on $\mathbb{C}^{2}$ is generated by $z_{1} \rightarrow e^{2 \pi i / r} z_{1}, z_{2} \rightarrow e^{-2 \pi i / r} z_{2}$. Dividing by this action produces an $A_{r-1}$ singularity, corresponding in seven dimensions to $S U(r)$ gauge symmetry.

Thus along $\mathbb{R}^{6} \times \mathbb{S}^{1}$, we have locally an $S U(r)$ gauge symmetry. All told, from a six-dimensional point of view, we have an $\mathbb{S}^{1}$ family of $A_{r-1}$ singularities. To determine the gauge group in six dimensions, the remaining question is to determine whether, in going around the circle, there is a monodromy that breaks $S U(r)$ to a subgroup. Such a monodromy would necessarily involve an outer automorphism of $S U(r)$; there is only one non-trivial outer automorphism, "complex conjugation." 3

In general, for a family of $A-D-E$ singularities, there is monodromy, breaking the gauge symmetry to a subgroup. This has been found to play an important role in $F$-theory $[12,13]$, and we will meet examples of such monodromy later. In the present case, however, the monodromy vanishes and the gauge group in six dimensions is actually $S U(r)$. This assertion can be justified as follows.

$\mathbb{C}^{2} / \mathbb{Z}_{r}$, regarded as a flat hyper-Kahler manifold with an orbifold singularity, actually has a $U(2)$ symmetry group. Part of the $U(2)$ is obscured when one picks a particular complex structure (as we have done in selecting complex coordinates $z_{1}, z_{2}$ to identify the flat hyper-Kahler manifold $\mathbb{R}^{4}$ with $\mathbb{C}^{2}$ ), but part of it is still manifest. In particular, there is an evident $U(1)$ group of symmetries, which we will call $F$ :

$$
\begin{aligned}
& z_{1} \rightarrow e^{i \alpha} z_{1} \\
& z_{2} \rightarrow e^{-i \alpha} z_{2} .
\end{aligned}
$$

Since this group is connected, and $S U(r)$ has no non-trivial outer automorphisms that can be continuously connected to the identity, $F$ simply commutes with the $S U(r)$ gauge symmetry. ${ }^{4}$ Now, the particular family of

\footnotetext{
${ }^{3}$ Monodromy consisting of an inner automorphism, that is an element of $S U(r)$, means that there is a Wilson line on $\mathbb{S}^{1}$ breaking $S U(r)$ to a subgroup. One can always turn on such a Wilson line before shrinking the radius of $\mathbb{S}^{1}$, but this does not give anything essentially new in the following sense. As in the discussion of (3.3), this gives the same six-dimensional theory that one would obtain by first taking the small radius limit with unbroken $S U(r)$ and then Higgsing the six-dimensional theory.

${ }^{4}$ This argument should really be made in two steps. (1) As there are no connected outer automorphisms of $S U(r)$, the automorphism of $S U(r)$ brought about by $F$ is inner. Its generator is hence the sum of a quantity that commutes with $S U(r)$ and an $S U(r)$ generator. (2) Hence, by defining correctly the $F$ action on degrees of freedom localized near the origin (to remove the possible $S U(r)$ generator from the generator of $F$ ), $F$ can be defined so as to commute with $S U(r)$.
} 
$A_{r-1}=\mathbb{C}^{2} / \mathbb{Z}_{r}$ singularities of interest here can be described by saying that as one goes around the $\mathbf{S}^{1}, \mathbb{C}^{2} / \mathbb{Z}_{r}$ is transformed by a $\mathbb{Z}_{q}$ transformation. The monodromy of interest is determined by the action of $\mathbb{Z}_{q}$ on the $S U(r)$ gauge symmetry. (Only the quotient $\mathbb{Z}_{s}=\mathbb{Z}_{q} / \mathbb{Z}_{r}$ acts faithfully on $\mathbb{C}^{2} / \mathbb{Z}_{r}$.) In view of the form of 3.4, $\mathbb{Z}_{q}$ acts on $\mathbb{C}^{2}$ as a subgroup of $F$, and hence commutes with $S U(r)$ and, as we claimed, does not generate a non-trivial outer automorphism of $S U(r)$.

Therefore, we have obtained a family of exotic six-dimensional theories, with the same $(1,1)$ supersymmetry, the same low energy gauge group, and parametrized by the same pairs $(p, q)$, as for the $(p, q)$ fivebrane system of Type IIB. This strongly suggests that the two classes of six-dimensional theories are the same. In the next section, we give via $T$-duality further evidence for this interpretation.

\section{$4 \quad T$-Duality and $(p, q)$ Fivebrane System}

First we recall the analysis via $T$-duality of the relation between Type IIA at an $A_{q-1}$ singularity and the $(0, q)$ fivebrane system. A useful reference for this discussion is [10].

Applying $T$-duality directly to $\mathbb{C}^{2} / \mathbb{Z}_{q}$ is inconvenient. It requires picking one of the $U(1)$ symmetries of $\mathbb{C}^{2} / \mathbb{Z}_{q}$ and applying $T$-duality on the orbits. The radius of the orbits grows at infinity, so the $T$-dual has orbits whose size vanishes at infinity; its behavior at infinity is difficult to interpret.

However, the six-dimensional theory obtained from weakly coupled Type IIA at an $A_{q-1}$ singularity is independent of the global structure of $\mathbb{C}^{2} / \mathbb{Z}_{q}$ and depends only on the behavior near the singularity. It is hence much more convenient to imbed the singularity in a four-manifold which has a convenient $\mathbb{S}^{1}$ symmetry with a circle of fixed radius at infinity. Such a solution is the multi Kaluza-Klein monopole solution:

$$
d s^{2}=U\left(d y-A_{i} d x^{i}\right)^{2}+U^{-1}(d \vec{x})^{2},
$$

with

$$
U=\left(1+\sum_{a=1}^{q} \frac{S}{2\left|\vec{x}-\vec{x}_{a}\right|}\right)^{-1}
$$

Here $\vec{x}$ is a three-vector, $\vec{x}_{a}$ are the monopole positions for $a=1, \ldots, q$, $A=A_{i} d x^{i}$ is the vector potential due to $q$ Dirac monopoles at the given points, and $y$ is a periodic variable of period $2 \pi S$. In the special case that the $\vec{x}_{a}$ all coincide, the solution (4.1) has an $A_{q-1}$ singularity at $\vec{x}=\vec{x}_{a}$, where also $U=0$. Near infinity it looks like an $\mathbb{S}^{1}$ bundle over $\mathbb{R}^{3}$ (with $y$ 
and $\vec{x}$ being coordinates of $\mathbb{S}^{1}$ and $\mathbb{R}^{3}$, respectively), with first Chern class $q$.

This solution has the interpretation of a system of $q$ Kaluza-Klein monopoles on $\mathbb{R}^{3} \times \mathbb{S}^{1}$. The presence of the monopoles causes a topological twisting at infinity. The $\mathbb{S}^{1}$ can be seen in the solution (4.1) as the orbits of the $U(1)$ symmetry that acts by $y \rightarrow y+$ constant. We will call this symmetry $F$ (near the origin it looks just like the symmetry called $F$ in the previous section).

Now make a $T$-duality transformation, on the $F$ orbits, to convert to a Type IIB description. The $T$-duality converts Kaluza-Klein monopoles to NS fivebranes, as one can see by looking at how $T$-duality acts on the fields that the monopoles and fivebranes create at infinity [14]. ${ }^{5}$ So the Type IIB dual of the multi-Kaluza-Klein monopole solution is a configuration of $q$ NS fivebranes on $\mathbb{R}^{3} \times \mathbb{S}^{1}$. The explicit solution of the low energy field equations describing $q$ NS fivebranes on $\mathbb{R}^{3} \times \mathbb{S}^{1}$ is known in closed form, but will not be needed here.

From $q$ NS fivebranes on $\mathbb{R}^{3} \times \mathbb{S}^{1}$, one gets a $S U(q)$ gauge symmetry when and only when the fivebranes coincide in space. Type IIA on a multiKaluza-Klein monopole spacetime has extended gauge symmetry when and only when the $\vec{x}_{a}$ coincide, at which point there is an $A_{q-1}$ singularity. So the enhanced gauge symmetry from an $A_{q-1}$ singularity must be mapped to the enhanced gauge symmetry from coincident Type IIB fivebranes. ${ }^{6}$

Upon taking the limit as the string coupling constant goes to zero, it follows that the decoupled six-dimensional theory obtained from an $A_{q-1}$ singularity in Type IIA is the same as the decoupled six-dimensional theory obtained from coincident NS fivebranes in Type IIB.

We can now carry out a discussion analogous to that of (3.3). The configuration space of $q$ NS fivebranes on $\mathbb{R}^{3} \times \mathbb{S}^{1}$ is $\left(\mathbb{R}^{3} \times \mathbb{S}^{1}\right)^{q} / \mathbb{S}_{q}$. After factoring out the decoupled center of mass position, the moduli space for the relative or internal motion is $\left(\mathbb{R}^{3} \times \mathbb{S}^{1}\right)^{q-1} / \mathbb{S}_{q}$. However, in the limit that the Type IIB string coupling is taken to zero, the radius of the $\mathbb{S}^{1}$ diverges

\footnotetext{
${ }^{5}$ This analysis proceeds as follows. In compactification on a circle, one generates in Type II superstring theory two $U(1)$ gauge fields; one comes from the metric tensor and one from the two-form field $B$. $T$-duality exchanges these two gauge fields (which couple to string momentum and winding, respectively). Hence it exchanges the Kaluza-Klein magnetic charge - which is the magnetic charge of the $U(1)$ that comes from the metric with a flux of $H=d B$. This flux can, of course, be seen in the explicit asymptotic formula for the $T$-dual of the Kaluza-Klein monopole. But the $H$ flux is the NS fivebrane number. Hence, the $T$-dual of a Type IIA state with Kaluza-Klein monopole charge $q$ is a Type IIB state with NS fivebrane charge $q$.

${ }^{6} \mathrm{On}$ each side there is an additional $U(1)$ that is decoupled from the $S U(q)$. In the Type IIB description it comes from the center of mass motion of the $q$ fivebranes, and in Type IIA it comes from a zero mode of the $B$ field that is not supported near the singularity .
} 
in the relevant units. ${ }^{7}$ The six-dimensional theory obtained by taking the coupling to zero while sitting at some point $P \in\left(\mathbb{R}^{3} \times \mathbb{S}^{1}\right)^{q-1} / \mathbb{Z}_{q}$ only "sees" a small neighborhood of $P$. The most interesting theory, with low energy gauge group $S U(q)$, is obtained by taking $P$ to be the most singular point at which all fivebranes coincide in space. All possibilities can be obtained by Higgsing this theory.

\section{$(p, q)$ Generalization}

Our goal is to consider the $(p, q)$ case in a similar way.

Let $W$ be the charge one Kaluza-Klein monopole solution:

$$
d s^{2}=U\left(d y-A_{i} d x^{i}\right)^{2}+U^{-1}(d \vec{x})^{2}
$$

with

$$
U=\left(1+\frac{S}{2|\vec{x}|}\right)^{-1}
$$

$y$ is a periodic variable of period $2 \pi S ; W$ is a smooth manifold. Consider the $\mathbb{Z}_{q}$ action on this space generated by $y \rightarrow y+2 \pi S / q$. The quotient $W_{q}=W / \mathbb{Z}_{q}$ is a singular hyper-Kahler manifold with an $A_{q-1}$ orbifold singularity at the origin; it is actually equivalent to the special case of the multi-Kaluza-Klein monopole solution (4.1) in which the monopoles are all coincident at the origin (but with $S$ replaced by $S / q$ ).

We want to consider the same construction as in section 3 , but with $\mathbb{C}^{2}$ replaced by $W$ and $\mathbb{C}^{2} / \mathbb{Z}_{q}$ replaced by $W_{q}$. The change is inessential as far as the limiting six-dimensional theories are concerned (since $W$ and $W / \mathbb{Z}_{q}$ look near the origin like $\mathbb{C}^{2}$ and $\left.\mathbb{C}^{2} / \mathbb{Z}_{q}\right)$ but makes it possible to use $T$-duality. So we let $\mathbb{S}^{1}$ be a circle with periodic coordinate $t$ of period $2 \pi R$, and we consider $M$-theory on a quotient $Y_{p, q}=\left(W \times \mathbb{S}^{1}\right) / \mathbb{Z}_{q}$, with $\mathbb{Z}_{q}$ acting on $W$ as in the last paragraph and on $\mathbb{S}^{1}$ by $t \rightarrow t-2 \pi R(p / q)$.

We can now make an argument just like that in section 3 . In the limit of $R \rightarrow 0, M$-theory on $\mathbb{R}^{6} \times Y_{p, q}$ looks like free Type IIA superstring theory, everywhere except near fixed points of the $\mathbb{Z}_{q}$ action on $W$ (that is, near $\vec{x}=0$ in (4.3)). Near those fixed points, $Y_{p, q}$ looks like the space $X_{p, q}=$ $\left(\mathbb{C}^{2} \times \mathbb{S}^{1}\right) / \mathbb{Z}_{q}$ studied in section 3 . The surviving six-dimensional theory in the limit $R=0$ should be the same whether one considers $X_{p, q}$ or $Y_{p, q}$. But (as in the $p=0$ case that was reviewed above) using $Y_{p, q}$ makes it easier to make a relation to Type IIB.

\footnotetext{
${ }^{7}$ The reason for this is that as the NS fivebrane has a tension of order $1 / \lambda^{2}$, in order to have energies of order 1 its transverse fluctuations have a length scale, in string units, of order $\lambda$. After rescaling units so that the transverse fluctuations are of order one, the $\mathbb{S}^{1}$ factor in the moduli space (which in string units has a size of order 1 ) is of order $1 / \lambda$. For related issues concerning fivebranes with transverse circles, see [15].
} 
The relation to Type IIB is made by using the fact that $M$-theory on $\mathbf{T}^{2}$ is equivalent to Type IIB on $\mathbb{S}^{1}$. Let $N$ be the quotient of $W$ by the $U(1)$ symmetry $y \rightarrow y+$ constant, which we have called $F$. Thus, $N$ is a copy of $\mathbb{R}^{3}$, parametrized by $\vec{x}$, but actually has a singularity at $\vec{x}=0$. Near infinity, $W$ is fibered over $N$ with $\mathbb{S}^{1}$ fibers of circumference $2 \pi S$. Hence $W \times \mathbb{S}^{1}$ is fibered over $N$ with fibers $\mathbf{T}^{2}=\mathbb{S}^{1} \times \mathbb{S}^{1}$; metrically the $\mathbf{T}^{2}$ is a rectangular torus that is a product of circles with circumferences $2 \pi S$ and $2 \pi R$. One can think of $\mathbf{T}^{2}$ as the quotient of the $y-t$ plane by the lattice $\Gamma$ generated by $e=(2 \pi S, 0)$ and $f=(0,2 \pi R)$. The $\tau$ parameter of this $\mathbf{T}^{2}$ is hence

$$
\tau=i \frac{S}{R}
$$

and as $R \rightarrow 0, M$-theory on $W \times \mathbb{S}^{1}$ is equivalent near infinity to weakly coupled Type IIB on $N \times \mathbb{S}^{1}$. (The $\mathbb{S}^{1}$ in this Type IIB description is a dual circle with a radius of order $1 / R S$.) Near the origin in $N$, there is a singularity of some kind in the Type IIB theory. From our above discussion of $T$-duality in Type IIA on $W$ (which is the same as $M$-theory on $W \times \mathbb{S}^{1}$ ), this singularity is an NS fivebrane.

Now we want to repeat this for $M$-theory on $Y_{p, q}=\left(W \times \mathbb{S}^{1}\right) / \mathbb{Z}_{q}$. This still looks at infinity like a two-torus bundle over $N$. The fibers are now copies of $\widetilde{T}=\mathbf{T}^{2} / \mathbb{Z}_{q}$, where $\mathbb{Z}_{q}$ acts by $y \rightarrow y+2 \pi S / q, t \rightarrow t-2 \pi R(p / q)$. $\widetilde{T}$ is the torus obtained by dividing the $y-t$ plane by the lattice $\widetilde{\Gamma}$ generated by $f=(0,2 \pi R)$ and $\widetilde{e}=(2 \pi S / q,-2 \pi R p / q)$. The $\tau$ parameter of this lattice is

$$
\tau=i \frac{S}{q R}-\frac{p}{q},
$$

and this is the $\tau$-parameter of the equivalent Type IIB theory. In particular, for $R \rightarrow 0$ we get as before a weakly coupled Type IIB theory, but now with a theta angle $\theta_{B}=-2 \pi p / q$. The significance, if any, of this theta angle will be the subject of the next section.

One has, evidently,

$$
e=q \widetilde{e}+p f .
$$

This implies the following. Consider Type IIB theory with a $\tau$ parameter associated with a lattice $\mathbb{R}^{2} / \Gamma$. The possible five-brane (or one-brane) charges correspond to lattice points in $\Gamma$. Five-brane (or one-brane) tensions are proportional to the lengths of the corresponding lattice vectors. For $M$-theory on $W \times \mathbb{S}^{1}$ with large $S / R$ interpreted as a weakly coupled Type IIB theory, the lattice vectors $e$ and $f$ correspond respectively to an NS fivebrane and a Dirichlet fivebrane. This spacetime has a charge corresponding to a single NS fivebrane (as we recalled above via $T$-duality from Type IIA), which 
corresponds to the charge vector $e$. After dividing by $\mathbb{Z}_{q}$, the charge vector $e$ is reexpressed in the lattice appropriate to $M$-theory on $\left(W \times \mathbb{S}^{1}\right) / \mathbb{Z}_{q}$ via (4.7). ${ }^{8}$ It therefore corresponds to a $(p, q)$ fivebrane system of Type IIB.

This strongly supports the claim that the six-dimensional theory extracted from $M$-theory on $X_{p, q}$ coincides with that obtained from a $(p, q)$ fivebrane system in weakly coupled Type IIB.

\section{The $\theta$ Angle}

An interesting feature of the $T$-duality argument made in the last section is that, in this approach, the Type IIB theory naturally appeared with a theta angle of $\theta_{B}=2 \pi \operatorname{Re} \tau=-2 \pi p / q$. Is this significant? This is a special case of the following question: does the physics of the six-dimensional theories obtained by taking the Type IIB coupling to zero depend on the Type IIB theta angle?

We will argue, though not conclusively, that the answer is "no." On the other hand, we will claim that these theories do have an observable and significant theta angle that depends on $p$ and $q$; it simply is not the Type IIB theta angle!

In the discussion so far, for each $r(r \geq 2)$, we have obtained infinitely many six-dimensional theories with low energy $S U(r)$ gauge group. Indeed, let $(a, b)$ be any pair of relatively prime integers; upon setting $(p, q)=(a r, b r)$ and considering a $(p, q)$ fivebrane system, we get for each pair $(a, b)$ a theory with low energy gauge group $S U(r)$. How would a low energy observer distinguish these theories? We will consider the case $r \geq 3$ (though the question is natural for $r=2$, and even for $r=1$ where there is no low energy gauge symmetry).

The low energy interactions are dominated by the $F^{2}$ term in the effective Lagrangian and its supersymmetric generalization; by considering only these interactions, one certainly cannot distinguish different theories with the same low energy gauge group. It will be necessary to look at interactions of higher dimension.

One higher dimension interaction of special significance is the theta angle of the gauge theory. For $r>2$, one has $\pi_{5}(S U(r))=\mathbb{Z}$, so there is a theta angle in $S U(r)$ gauge theory in six-dimensions. It could be measured, in principle, by a low energy observer studying the $(p, q)$ six-dimensional theory. (Its qualitative consequences are most readily understood in a topologically non-trivial situation, either on a general six-manifold or in the presence of

\footnotetext{
${ }^{8}$ In general dividing by $\mathbb{Z}_{q}$ does not commute with quantum dynamics, but since the charge is determined by the asymptotic "twisting" of the $\mathbf{T}^{2}$ at infinity, which is a topological invariant, the charge vector on $\left(W \times \mathbb{S}^{1}\right) / \mathbb{Z}_{q}$ is simply that of $W \times \mathbb{S}^{1}$ expressed in the new lattice.
} 
instanton strings on $\mathbb{R}^{6}$.) The question arises of what value of $\theta$ would be observed by a low energy observer probing the six-dimensional theory extracted from the $(p, q)$ system.

To answer this, we first consider the case of $r$ Dirichlet five-branes. This $(r, 0)$ case does not lead to an interacting six-dimensional theory in the limit that the Type IIB coupling vanishes, but of course there is a low-energy $S U(r)$ gauge symmetry for such a system. Moreover, according to the general theory of couplings of RR fields to $D$-brane world-volume gauge fields, for the $(r, 0)$ system, the space-time theta angle is simply equal to the underlying Type IIB theta angle. In fact, if $\beta$ is the RR scalar of Type IIB (so that $\theta_{B}=2 \pi \operatorname{Re} \tau$ is the expectation value of $\beta$ ), then there is a world-volume coupling $\beta \operatorname{Tr} F \wedge F \wedge F$, which upon setting $\beta$ to its vacuum expectation value reduces to

$$
\operatorname{Re} \tau \operatorname{Tr} F \wedge F \wedge F
$$

The coefficient of this interaction is precisely such ${ }^{9}$ that the space-time theta angle is $\theta=2 \pi \operatorname{Re} \tau$ for this case.

We can map the $(r, 0)$ system to a $(p, q)=(a r, b r)$ system via an $S L(2, \mathbb{Z})$ transformation by a matrix $\left(\begin{array}{ll}a & e \\ b & f\end{array}\right)$ for some $e, f$. (Suitable $e$ and $f$ exist because $a$ and $b$ are relatively prime.) This maps

$$
\tau \rightarrow \tau^{\prime}=\frac{a \tau+e}{b \tau+f}
$$

To get a decoupled six-dimensional theory, we need $\operatorname{Im} \tau^{\prime} \rightarrow \infty$, and this evidently happens for $\tau \rightarrow-f / b$. Since the $\theta$ angle is $2 \pi \operatorname{Re} \tau$, we get $\theta=$ $-2 \pi(f / b)$. (The sign actually depends on conventions that we will not try to fix precisely.)

Thus a low energy observer can distinguish the different theories with the same gauge group by measuring $\theta$. Now the question arises of whether only rational values of $\theta$ are possible in the low energy theory, or whether a generalization exists with irrational $\theta$. A proposal for a generalization of the construction discussed in the present paper to get variable $\theta$ has been made recently by Kol [17].

Note that the $\theta$ angle of the low energy theory depends only on the fivebrane charges, and not on an additional parameter such as $\operatorname{Re} \tau^{\prime}$. This gives some evidence that $\operatorname{Re} \tau^{\prime}$ is not an observable parameter in the theory obtained by taking $\operatorname{Im} \tau^{\prime} \rightarrow \infty$.

\footnotetext{
${ }^{9}$ The $\mathrm{RR}$ interactions are written as $\beta \mathrm{ch} F$, where ch is the Chern character [16]. The six-form part of the Chern character integrates in general for a gauge field of finite action (or on a compact six-manifold) to an arbitrary integer. That is why the normalization of the $\operatorname{Tr} F \wedge F \wedge F$ term precisely leads to $\theta=2 \pi \operatorname{Re} \tau$ with no additional numerical factor.
} 


\section{Other Gauge Groups}

We would like to generalize this discussion to consider six-dimensional theories with gauge groups other than $S U(n)$. We begin by analyzing theories with orthogonal and symplectic gauge groups, followed by a brief discussion of exceptional groups.

\subsection{Orientifolds and Their Cousins}

First we recall how orthogonal and symplectic gauge groups are obtained in Type IIB by using Dirichlet fivebranes. The basic idea is to consider such fivebranes at an orientifold sixplane. Thus, we consider Type IIB on $\mathbb{R}^{4} / \rho \Omega$, where $\rho$ is a reflection of all four coordinates, and the action of $\rho$ is combined with exchange of world-sheet left and right-movers, which we call $\Omega$. Away from the origin, $\mathbb{R}^{4} / \rho \Omega$ looks just like $\mathbb{R}^{4} / \rho$, but the behavior near the origin is different.

Since $\Omega$ reverses the sign of $\theta_{B}$, orientifolding of Type IIB can conceivably be carried out either at $\theta_{B}=0$ or at $\theta_{B}=\pi$. However, only the constructions at $\theta_{B}=0$ are well-understood. At $\theta_{B}=0$, there are two kinds of orientifold sixplane for Type IIB. They differ by the sign of the $\mathbb{R P}^{2}$ contribution in string perturbation theory. We will call the two types of sixplane $\mathcal{O}^{+}$and $\mathcal{O}^{-}$. If one brings $n$ Dirichlet fivebranes to the fixed point at the origin in $\mathbb{R}^{4},{ }^{10}$ then the action of $\Omega$ on the Chan-Paton factors is symmetric or anti-symmetric in the two cases, leading to orthogonal gauge groups for $\mathrm{O}^{+}$ and symplectic ones for $\mathrm{O}^{-}$. For $\mathrm{O}^{+}$, the number of fivebranes can be either even or odd, giving an $S O(n)$ gauge group with even or odd $n$. Fivebranes can only move to or from the origin in pairs, so the number of fivebranes at the origin is conserved modulo two. For $\mathcal{O}^{-}$, the number of fivebranes must be even, $n=2 m$, and the gauge group is $S p(m)$.

The orientifold six-planes carry Dirichlet fivebrane charge -2 in the case of $\mathcal{O}^{+}$, and +2 in the case of $\mathcal{O}^{-}$. The charge is reversed in going from $\mathcal{O}^{+}$to $\mathcal{O}^{-}$because the sign of the $\mathbb{R P}^{2}$ contribution to the world-sheet path integral is reversed. ${ }^{11}$

From Dirichlet fivebranes one cannot make a decoupled six-dimensional theory. To do so, we should consider NS fivebranes. So we make an $S$-duality transformation $\tau \rightarrow-1 / \tau$, which converts the D fivebranes to NS fivebranes, and also maps $\Omega$ to $(-1)^{F_{L}}$ - the operation that counts left-moving worldsheet fermions modulo two. The space-time is now $\mathbb{R}^{4} / \rho(-1)^{F_{L}}$; actually, there are necessarily two versions $\mathcal{U}^{ \pm}$of $\mathbb{R}^{4} / \rho \cdot(-1)^{F_{L}}$, obtained by $S$-duality

\footnotetext{
${ }^{10}$ I count the number of fivebranes as measured on the covering space $\mathbb{R}^{4}$; on $\mathbb{R}^{4} / \rho \Omega$ one sees half as many.

${ }^{11}$ The numerical value is obtained as follows. In general, an orientifold $10-k$-plane has $9-k$-brane charge $\mp 32\left(2^{-k}\right)$; this is $\mp 2$ for six-planes.
} 
from $\mathcal{O}^{+}$and $\mathcal{O}^{-}$, respectively. They have NS fivebrane charges of -2 and +2 , respectively. When $n$ NS fivebranes approach the origin in $\mathbb{R}^{4} / \rho \cdot(-1)^{F_{L}}$, one gets a gauge group $S O(n)$ or $S p(n / 2)$ for $\mathcal{U}^{+}$or $\mathcal{U}^{-}$. In the limit that the Type IIB string coupling constant $\lambda$ vanishes, one gets six-dimensional theories with these gauge groups, decoupled from the bulk.

In comparing to $M$-theory, it will be more convenient to replace $\mathbb{R}^{4} / \rho(-1)^{F_{L}}$ by $\left(\mathbb{R}^{3} \times \mathbb{S}^{1}\right) / \rho(-1)^{F_{L}}$ (where now $\rho$ acts as -1 on all three coordinates of $\mathbb{R}^{3}$ and also on an angular coordinate on $\left.\mathbb{S}^{1}\right)$. The reason, as in section 4 , is that this facilitates arguments using standard dualities. Since there are two fixed points in the $\rho$ action on $\mathbb{R}^{3} \times \mathbb{S}^{1}$, there are several distinct cases; the fixed points may be $\mathcal{U}^{+} \mathcal{U}^{+}, \mathcal{U}^{+} \mathcal{U}^{-}$, or $\mathcal{U}^{-} \mathcal{U}^{-}$. To obtain a decoupled six-dimensional theory, one takes $\lambda \rightarrow 0$, keeping the radius of the $\mathbb{S}^{1}$ fixed in string units. As in a footnote in section 4 , the scale of variation of the fivebrane positions is of order $\lambda$, so for $\lambda \rightarrow 0$, one does not "see" the whole $\mathbb{S}^{1}$, but only a vanishingly small neighborhood of the vacuum, which is determined by the fivebrane positions. Hence, in general inequivalent sixdimensional theories can be reached by taking $\lambda \rightarrow 0$ with different fivebrane positions; these six-dimensional theories will be essentially different in the sense that no one of them can be obtained by Higgsing another. The main examples are as follows. By $n$ fivebranes at a generic point, one gets for $\lambda \rightarrow 0$ the $U(n)$ theory studied above, which we will call $P_{n}$; from $n$ fivebranes at a $\mathcal{U}^{+}$fixed point, one gets an $S O(n)$ theory that we will call $Q_{n}$; from $n=2 m$ fivebranes at a $\mathcal{U}^{-}$point, we get an $S p(m)$ theory that we will call $R_{m}$. Other examples are simple consequences of these.

Here are some cases whose $M$-theory counterparts we will find later. $n$ will denote the total number of fivebranes. We recall that the number of fivebranes at a $\mathcal{U}^{+}$fixed point can be even or odd, and is conserved modulo two. In the following examples, there are several maximally singular configurations, so in the weak coupling limit, one can obtain distinct sixdimensional theories that cannot be Higgsed to one another.

(1) In the $\mathcal{U}^{+} \mathcal{U}^{+}$case, if $n$ is even and the number of fivebranes at each $\mathcal{U}^{+}$is even, then by placing $2 s$ fivebranes at one fixed point and $n-2 s$ at the other, we make for $\lambda \rightarrow 0$ the product theory $Q_{2 s} \times Q_{n-2 s}$ with low energy gauge group $S O(2 s) \times S O(n-2 s)$. No one of these theories can be Higgsed to another. Note that the total NS fivebrane charge of the orientifolds is -4 , so the total fivebrane charge is $n-4$.

(2) In the $\mathcal{U}^{-} \mathcal{U}^{+}$case, by placing $2 s$ fivebranes at $\mathcal{U}^{-}$and $n-2 s$ at $\mathcal{U}^{+}$, we make the theory $R_{s} \times Q_{n-2 s}$ with gauge group $S p(s) \times S O(n-2 s)$; these theories cannot be Higgsed to one another. The total NS fivebrane charge of the orientifolds is 0 , so the total fivebrane charge is $n$. This example makes sense whether $n$ is even or odd.

(3) In the $\mathcal{U}^{+} U^{+}$case, if $n$ is even and the number of fivebranes at each 
$\mathcal{U}^{+}$is odd, then by placing $2 s+1$ at one fixed point and $n-2 s-1$ at the other, we make the theory $Q_{2 s+1} \times Q_{n-2 s-1}$ with gauge group $S O(2 s+1) \times$ $S O(n-2 s-1)$; these theories cannot be Higgsed to one another. The total NS fivebrane charge of the orientifolds is -4 , so the total fivebrane charge is $n-4$.

\subsection{Approach via $M$-Theory}

Now we will approach the same subject via $M$-theory.

To start with, we recall how to get in seven dimensions a gauge group $D_{k}=S O(2 k)$. For this, one considers $M$-theory on $\mathbb{R}^{7} \times \mathbb{C}^{2} / \Gamma$, where $\Gamma$ is generated by group elements $\alpha, \beta$, obeying

$$
\begin{aligned}
\alpha^{2} & =\beta^{k-2} \\
\alpha \beta & =\beta^{-1} \alpha \\
\alpha^{4} & =\beta^{2 k-4}=1 .
\end{aligned}
$$

It follows from the first relation that $\alpha^{2}$ commutes with both $\alpha$ and $\beta$ and hence is a central element of the group, whose square is the identity according to the last relation. (The quotient of $\Gamma$ by the group generated by $\alpha^{2}$ is a dihedral group.)

The action of $\Gamma$ on $\mathbb{C}^{2}$ is

$$
\begin{aligned}
& \alpha:\left(\begin{array}{l}
z_{1} \\
z_{2}
\end{array}\right) \rightarrow\left(\begin{array}{c}
z_{2} \\
-z_{1}
\end{array}\right) \\
& \beta:\left(\begin{array}{c}
z_{1} \\
z_{2}
\end{array}\right) \rightarrow\left(\begin{array}{c}
e^{\pi i /(k-2)} z_{1} \\
e^{-\pi i /(k-2)} z_{2}
\end{array}\right) .
\end{aligned}
$$

We can obtain an $S O(2 k)$ gauge theory in six dimensions by considering $M$ theory on $\mathbb{R}^{6} \times \mathbb{C}^{2} / \Gamma \times \mathbb{S}^{1}$, where $\mathbb{S}^{1}$ has circumference $2 \pi R$. This theory is in fact equivalent to Type IIA on $\mathbb{R}^{6} \times \mathbb{C}^{2} / \Gamma$. The Type IIA string coupling constant vanishes for $R \rightarrow 0$, so the theory becomes free in bulk, but the $S O(2 k)$ gauge coupling, if expressed in string units, is non-zero in this limit. ${ }^{12}$ So the limiting theory is a six-dimensional theory with a low energy gauge group $S O(2 n)$.

As in section 3 , we will generalize $\mathbb{C}^{2} / \Gamma \times \mathbb{S}^{1}$ to $\left(\mathbb{C}^{2} \times \mathbb{S}^{1}\right) / \Gamma$, where $\Gamma$ acts as in 6.2 on $\mathbb{C}^{2}$ while also acting on $\mathbb{S}^{1}$. Because in the present paper, we wish to consider only theories with $(1,1)$ supersymmetry in six dimensions, we restrict ourselves to the case that $\Gamma$ acts on $\mathbb{S}^{1}$ by rotations (inclusion of reflections would break half the supersymmetry). Since the group of

\footnotetext{
${ }^{12}$ For example, gauge fields that gauge the maximal torus of $S O(2 k)$ are $\mathrm{RR}$ gauge fields of the perturbative Type IIA theory, and their gauge couplings are independent of the string coupling constant [18].
} 
rotations of $\mathbb{S}^{1}$ is abelian, $\alpha$ and $\beta$ commute as rotations, and hence the relation $\alpha \beta=\beta^{-1} \alpha$ reduces to $\beta^{2}=1$.

There hence are two types of examples:

(A) $\beta$ can act trivially on $\mathbb{S}^{1}$, in which case $\alpha^{2}=1$, so $\alpha$ is a rotation by either zero or $\pi$.

(B) $\beta$ may act by rotation by $\pi$, in which case the relation $\alpha^{2}=\beta^{k-2}$ means that $\alpha$ is a rotation by 0 or $\pi$ if $k$ is even, or by $\pm \pi / 2$ if $k$ is odd. Whether $k$ is even or odd, the two choices of $\alpha$ are equivalent, because $\alpha$ can be conjugated to $\alpha \beta$ by a transformation of the form $z_{1} \rightarrow e^{i \pi / 2(k-2)} z_{1}, z_{2} \rightarrow$ $e^{-i \pi / 2(k-2)} z_{2}$.

Either type of example can be given a Type IIB description by reasoning along the lines of section 4 . One first replaces $\mathbb{C}^{2}$ by the Kaluza-Klein monopole space $W$. $W$ has $S U(2)$ symmetry (which acts by rotation of $\vec{x}$ ), and the embedding of $\Gamma$ in $S U(2)$ enables us to view $\Gamma$ as a symmetry group of $W$. As such, $\Gamma$ acts freely except for a fixed point at $\vec{x}=0$, near which the action of $\Gamma$ on $W$ looks like its action on $\mathbb{C}^{2}$. Thus, to study possible six-dimensional theories supported near the fixed point, one may replace $\mathbb{C}^{2}$ by $W$.

Having done so, we proceed as in section 4 . To construct $\left(W \times \mathbb{S}^{1}\right) / \Gamma$, we first divide $W \times \mathbb{S}^{1}$ by the group $\Gamma^{\prime}$ generated by $\beta$. This group is isomorphic to $\mathbb{Z}_{2 k-4}$. As seen in section $4, M$-theory on $\left(W \times \mathbb{S}^{1}\right) / \Gamma^{\prime}$ has an interpretation in terms of Type IIB compactification on $N \times \mathbb{S}^{1}(N=W / U(1)$ is a copy of $\mathbb{R}^{3}$, parametrized by $\vec{x}$, but with a singularity at the origin) with a theta angle that is $\theta_{B}=0$ in case (A) ( $\beta$ acts trivially on $\left.\mathbb{S}^{1}\right)$ or $\theta_{B}=\pi$ in case (B) ( $\beta$ generates a $\pi$ rotation of $\mathbb{S}^{1}$ ). If we divide only by $\Gamma^{\prime}$, we get a model that has a Type IIB description, explained in section 4 , with $2 k-4$ NS fivebranes and either 0 or $k-2 D$ fivebranes, for case (A) or case (B).

What about the effect of dividing by $\alpha$ ? A key point is that $\alpha$ anticommutes with the $U(1)$ symmetry, which we have called $F$, that generates the fibers of $W \rightarrow N$. Hence on the two-torus fibers $\mathbf{T}^{2}=\mathbb{S}^{1} \times \mathbb{S}^{1}$ of $W \times \mathbb{S}^{1}$ (as in section 4, the first factor in $\mathbf{T}^{2}$ is the orbit of $F$ and the second factor in $\mathbf{T}^{2}$ is the second factor in $W \times \mathbb{S}^{1}$ ), $\alpha$ reverses the orientation of the first factor and preserves the orientation of the second factor. $\alpha$ thus acts as a $2 \times 2$ matrix

$$
\left(\begin{array}{rr}
-1 & 0 \\
0 & 1
\end{array}\right)
$$

generating an outer automorphism of the $S L(2, \mathbb{Z})$ symmetry group of Type IIB string theory. In perturbative Type IIB string theory, this particular symmetry is seen as $(-1)^{F_{L}}$, the transformation that counts left-moving 
world-sheet fermions modulo two. ${ }^{13}$ Note that this transformation changes the sign of $\theta_{B}$ (while leaving fixed the string coupling constant), so it is a symmetry only in the two cases $\theta_{B}=0$ and $\theta_{B}=\pi$, the two values that we are actually encountering.

What about the action of $\alpha$ on the spacetime $N \times \mathbb{S}^{1}$ of the Type IIB description? $\alpha$ reverses the orientation of the $\mathrm{T}^{2}$ in the $M$-theory description, so (as $M$-theory membrane wrapping on $\mathbf{T}^{2}$ becomes momentum on $\mathbb{S}^{1}$ in Type IIB) it reverses the orientation of the $\mathbb{S}^{1}$. Since Type IIB does not admit orientation-reversing symmetries, $\alpha$ must also reverse the orientation of $N$. Since $\alpha$ must also commute with rotations of $\vec{x}$, it must act by $\vec{x} \rightarrow-\vec{x}$. (Of course, these assertions can also be verified directly, using the form of the Kaluza-Klein monopole solution).

Combining these results, $\alpha$ acts on the (A) models as a reversal of all four coordinates of $\mathbb{R}^{3} \times \mathbb{S}^{1}$, together with $(-1)^{F_{L}}$. Thus $\alpha$ is the transformation $\rho(-1)^{F_{L}}$ that we encountered in our Type IIB discussion above.

\section{Gauge Groups Of (A) Models}

Let us now determine, from an $M$-theory point of view, the low energy gauge groups of the (A) models. These are constructed from $M$-theory on $\mathbb{R}^{6} \times\left(W \times \mathbb{S}^{1}\right) / \Gamma$ (or simply $\left.\mathbb{R}^{6} \times\left(\mathbb{C}^{2} \times \mathbb{S}^{1}\right) / \Gamma\right)$ with $\alpha$ rotating the $\mathbb{S}^{1}$ by zero or $\pi$. Thus we consider two cases.

If the rotation angle is zero, this theory is simply $M$-theory on $\mathbb{R}^{6} \times$ $W / \Gamma \times \mathbb{S}^{1}$. This is equivalent to Type IIA on $\mathbb{R}^{6} \times W / \Gamma$, and has gauge group $S O(2 n)$ if the Wilson line around $\mathbb{S}^{1}$ is trivial. More generally, the Wilson line around $\mathbb{S}^{1}$ can break $S O(2 n)$ to a subgroup. Interesting special cases are that the Wilson line is a diagonal matrix with $2 s$ eigenvalues -1 and $2 n-2 s$ eigenvalues +1 . (The number of -1 's must be even, as the Wilson line is an element of $S O(2 n)$.) By picking this configuration and taking the limit $\lambda \rightarrow 0$, one obtains a six-dimensional theory with gauge group $S O(2 s) \times S O(2 n-2 s)$. Once one has flowed to a six-dimensional theory decoupled from the bulk, these theories cannot be Higgsed to one another (as the scalar fields are Lie algebra valued and not group-valued, or more rigorously the moduli are $\mathbb{R}$-valued and not $\mathbb{S}^{1}$-valued).

This theory should be dual to Type IIB on $\left(\mathbb{R}^{3} \times \mathbb{S}^{1}\right) / \rho(-1)^{F_{L}}$, with NS fivebrane charge $2 k-4$. (2k-4 is the fivebrane charge produced by dividing by the group $\Gamma^{\prime}$ generated by $\beta$; this group is isomorphic to $\mathbb{Z}_{2 k-4}$.) Making an $S$-duality transformation to $D$-fivebranes on $\left(\mathbb{R}^{3} \times \mathbb{S}^{1}\right) / \rho \Omega$, we conclude that the results found in the last paragraph should match one of the examples given at the end of section 6.1 with Dirichlet fivebrane charge

\footnotetext{
${ }^{13}$ The fact that the first eigenvalue of $\alpha$ is -1 and the second is +1 means that $\alpha$ reverses the sign of Dirichlet fivebrane charge and commutes with NS fivebrane charge, and so can be identified as $(-1)^{F_{L}}$.
} 
$2 k-4$. (Our analysis of the duality between $M$-theory and Type IIB involved mainly the behavior at infinity and was not precise enough to predict which kind of orientifold planes should be placed at the fixed points in the Type IIB description, so we must search by hand among the examples given in section 6.1.) Inspection shows that the $M$-theory results agree with example (1) at the end of section 6.1 if $n=2 k$. So we propose that these examples match, and as the most interesting consequence that the six-dimensional $S O(2 k)$ theories obtained from $M$-theory on $\mathbb{C}^{2} / \Gamma \times \mathbb{S}^{1}$ are the same as those obtained from Type IIB with $2 k$ NS fivebranes at a $\mathcal{U}^{+}$orientifold sixplane.

This essentially familiar result has been described in some detail to facilitate the analysis of subsequent examples. Let us now consider the second model of type (A), in which $\alpha$ acts by a $\pi$ rotation on $\mathbb{S}^{1}$.

Let as before $\Gamma^{\prime}$ be the subgroup of $\Gamma$ generated by $\beta$. Since $\alpha$ acts freely on $W \times \mathbb{S}^{1}$, singularities are generated only by the action of $\Gamma^{\prime}$. The origin in $W$ is an isolated fixed point for the action of $\Gamma^{\prime}$. As $\Gamma^{\prime}$ is isomorphic to $\mathbb{Z}_{2 k-4}, W / \Gamma^{\prime}$ has an $A_{2 k-5}$ singularity at the origin. Since $\Gamma^{\prime}$ acts trivially on $\mathbb{S}^{1},\left(W \times \mathbb{S}^{1}\right) / \Gamma^{\prime}=W / \Gamma^{\prime} \times \mathbb{S}^{1}$ has a circle of $A_{2 k-5}$ singularities. Locally along $\mathbb{S}^{1}$, there is therefore an $S U(2 k-4)$ gauge group. The fivebrane charge, produced by dividing $W$ by $\beta$ and then reinterpreting in Type IIB language, is $2 k-4$ as in the previous example.

However, once we divide also by $\alpha$, which rotates $\mathbb{S}^{1}$ and also acts by an automorphism of $W / \Gamma^{\prime}$, we get a non-trivial family of $A_{2 k-1}$ singularities fibered over $\mathbb{S}^{1}$. The question arises of whether the monodromy of this family acts by an outer automorphism of $S U(2 k-4)$. In fact, precisely this sort of monodromy of a family of $S U(2 n)$ singularities is encountered in $F$-theory, where it is important that this monodromy is indeed a non-trivial outer automorphism of $S U(2 k-4)[12,13]$. In fact, modulo inner automorphisms (which correspond to turning on Wilson lines), it is the unique outer automorphism of $S U(2 k-4)$; it acts by charge conjugation, and will be called $\Psi$.

When an $S U(2 k-4)$ group element $g$ is transported around the $\mathbb{S}^{1}$, it returns to $\Psi(g)=\bar{g}$ if there is no Wilson line on the $\mathbb{S}^{1}$. If on the other hand there is a Wilson line corresponding to an element $a \in S U(2 k-4),{ }^{14}$ then the monodromy is $g \rightarrow a \bar{g} a^{-1}$. The unbroken gauge group consists of group elements such that

$$
g=a \bar{g} a^{-1} .
$$

This unbroken gauge group depends on $a$. For $a=1$, the unbroken gauge group is $S O(2 k-4)$. Suppose on the other hand that $a$ is block diagonal,

\footnotetext{
${ }^{14}$ An interplay between Wilson lines and outer automorphisms similar to what follows has recently been uncovered in $F$-theory [19].
} 
with $s$ blocks that are copies of

$$
\left(\begin{array}{rr}
0 & 1 \\
-1 & 0
\end{array}\right)
$$

as well as a $2 k-4-2 s$-dimensional identity matrix. Then the unbroken gauge group is $S p(s) \times S O(2 k-4-2 s)$. These gauge groups, and the total fivebrane charge of $2 k-4$, agree with example (2) of section 6.1 if we set $n=2 k-4$.

The most interesting case is to set $s=k-2$. We learn that from $M$ theory on $\left(\mathbb{C}^{2} \times \mathbb{S}^{1}\right) / \Gamma$, in the limit that the radius of the $\mathbb{S}^{1}$ goes to zero, we can extract a six-dimensional theory with gauge group $S p(k-2)$, and that this is the same theory constructed in Type IIB from $2 k-4$ NS fivebranes at a singularity $\mathbb{R}^{4} / \rho(-1)^{F_{L}}$ of type $\mathcal{U}^{-}$.

\section{Mathematical Formulation}

Before going on, it will be useful to put the above discussion in a standard mathematical framework, as we will encounter several additional examples with the same basic structure. By mapping $\beta$ to the trivial rotation of $\mathbb{S}^{1}$ and $\alpha$ to a rotation by $\pi$, we have defined a homomorphism from $\Gamma$ to a subgroup $\mathbb{Z}_{2}$ of the group of rotations of $\mathbb{S}^{1}$. The kernel of this homomorphism is $\Gamma^{\prime}$, the group generated by $\beta$. Thus there is an exact sequence

$$
0 \rightarrow \Gamma^{\prime} \rightarrow \Gamma \rightarrow \mathbb{Z}_{2} \rightarrow 0 .
$$

In the $\Gamma$ action on $\mathbb{C}^{2} \times \mathbb{S}^{1}$, the subgroup $\Gamma^{\prime}$ acts only on $\mathbb{C}^{2}$, with a fixed point at the origin, and so generates a circle of fixed points in $\left(\mathbb{C}^{2} \times \mathbb{S}^{1}\right) / \Gamma$. The quotient $\mathbb{Z}_{2}$ acts freely on $\mathbb{C}^{2} \times \mathbb{S}^{1}$ (since it acts freely on $\mathbb{S}^{1}$ ). So the local structure is a singularity of type $\Gamma^{\prime}$. Globally, one has a circle of $\Gamma^{\prime}$ singularities, with a monodromy generated by the quotient $\mathbb{Z}_{2}$.

In generalizations, $\Gamma$ will be replaced by a possibly different finite subgroup of $S U(2), \Gamma^{\prime}$ will be a subgroup of $\Gamma, \mathbb{Z}_{2}$ will be replaced by a group $\mathbb{Z}_{n}$ of rotations of $\mathbb{S}^{1}$, for some $n$, and (6.6) will be replaced by an exact sequence

$$
0 \rightarrow \Gamma^{\prime} \rightarrow \Gamma \rightarrow \mathbb{Z}_{n} \rightarrow 0 .
$$

We will consider $M$-theory on $\left(\mathbb{C}^{2} \times \mathbb{S}^{1}\right) / \mathbb{Z}_{n}$, where $\Gamma^{\prime}$ acts only on $\mathbb{C}^{2}$, but $\Gamma$ acts also on $\mathbb{S}^{1}$ via the homomorphism to $\mathbb{Z}_{n}$. The singularity in $\left(\mathbb{C}^{2} \times \mathbb{S}^{1}\right) / \mathbb{Z}_{n}$ is a circle of $\Gamma^{\prime}$ singularities, with $\mathbb{Z}_{n}$ monodromies, just as in the above examples. The possibilities for $\Gamma, \Gamma^{\prime}$, and $n$ have in fact been classified by Reid in [20], p. 376. There are six examples, of which we have so far analyzed two. Reid's example (1) corresponds to the $(p, q)$ fivebrane system studied in sections 2-5, while his example (3) (with $n+2$ identified with what we have called $k$ ) is the one we have just examined. 
The (B) Models

As explained above, there is up to conjugation only one (B) model for each $k$, but the details depend somewhat on whether $k$ is even or odd. First we assume $k=2 p$ even. This is Reid's example (5).

In this case, we can suppose that $\alpha$ acts trivially on $\mathbb{S}^{1}$, while $\beta$ rotates $\mathbb{S}^{1}$ by $\pi$. The subgroup $\Gamma^{\prime}$ is hence generated by $\alpha$ and $\gamma=\beta^{2}$, with relations

$$
\begin{aligned}
\alpha^{2} & =\gamma^{p-1} \\
\alpha \gamma & =\gamma^{-1} \alpha \\
\alpha^{4} & =\gamma^{2 p-2} .
\end{aligned}
$$

$\Gamma^{\prime}$ is the finite subgroup of $S U(2)$ related to $D_{p+1}=S O(k+2)$. The action of $\Gamma$ on $\mathbb{C}^{2} \times \mathbb{S}^{1}$ is determined by an exact sequence

$$
0 \rightarrow \Gamma^{\prime} \rightarrow \Gamma \rightarrow \mathbb{Z}_{2} \rightarrow 0 .
$$

The global structure is thus a circle of $\mathbb{C}^{2} / \Gamma^{\prime}$ singularities, with $\mathbb{Z}_{2}$ monodromy. In a Type IIB description, the fivebrane charge, being produced by dividing by $\gamma$ (which generates a cyclic group of order $2 p-2$ ) is $2 p-2=k-2$.

The $\mathbb{Z}_{2}$ monodromy is modulo inner automorphisms the unique outer automorphism of $S O(k+2)$. One can take it to be generated by a reflection on one of the $k+2$ coordinates. With a suitable choice of Wilson line, this breaks $S O(k+2)$ to $S O(2 r+1) \times S O(k+1-2 r)$ (and various subgroups to which one of these can be Higgsed). These gauge groups, and the fivebrane charge, agree with example (3) at the end of section 6.1 if we set $n=k+2$.

We learn from this that a six-dimensional theory with gauge group $S O(2 r+1)$ can be obtained from a small radius limit of $M$-theory on $\left(W \times \mathbb{S}^{1}\right) / \Gamma$, with suitable Wilson lines, and that this theory coincides with what is obtained from Type IIB with $2 r+1$ NS fivebranes at a singularity $\mathbb{R}^{4} / \rho(-1)^{F_{L}}$ of type $\mathcal{U}^{+}$.

Finally, we consider the model of type (B) with $k$ odd, say $k=2 p+1$. This correspond to Reid's example (2). ${ }^{15}$ In this case, $\alpha$ is a $\pi / 2$ rotation. The group $\Gamma^{\prime}$ that acts trivially on $\mathbb{S}^{1}$ is generated by $\gamma=\beta^{2}$, with $\gamma^{2 p-1}=1$. The exact sequence is

$$
0 \rightarrow \Gamma^{\prime} \rightarrow \Gamma \rightarrow \mathbb{Z}_{4} \rightarrow 0
$$

where one can regard $\mathbb{Z}_{4}$ as the group generated by $\alpha$.

Since $\Gamma^{\prime}$ is isomorphic to $\mathbb{Z}_{2 p-1}$, the fivebrane charge in a Type IIB description is $2 p-1$. In $M$-theory on $\left(\mathbb{C}^{2} \times \mathbb{S}^{1}\right) / \Gamma$, the global structure is a circle of $A_{2 p-2}$ singularities with monodromies generated by $\mathbb{Z}_{4}$. Actually, $A_{2 p-2}$ is $S U(2 p-1)$, and the monodromy is such that the generator $\alpha$ of $\mathbb{Z}_{4}$ acts

\footnotetext{
${ }^{15} \mathrm{~A}$ misprint was pointed out by D. Morrison; $D_{2 n+1}$ should read $D_{2 n+3}$.
} 
by complex conjugation, while $\alpha^{2}$ induces a trivial monodromy. $\left(\mathbb{Z}_{4}\right.$ could not act faithfully, because the group of outer automorphisms of $S U(2 p-1)$ is $\mathbb{Z}_{2}$.)

As in (6.4), the unbroken gauge group depends on a Wilson line $a$. If $a$ is the sum of $s$ copies of the $2 \times 2$ matrix in (6.5) plus an identity matrix, then the unbroken gauge group is $S p(s) \times S O(2 p-1-2 s)$. These possibilities agree with example (2) in section 6.1 , with $n$ now being the odd number $n=2 p-1$. We conclude that $M$-theory on $\left(\mathbb{C}^{2} \times \mathbb{S}^{1}\right) / \Gamma$, with this $\Gamma$ action, can give, in a small radius limit with suitable Wilson lines, a variety of $S O$, $S p$, and $S U$ theories.

We thus obtain, in particular, a second construction of $S p(n)$ theories in six dimensions. Since $\pi_{5}(S p(n))=\mathbb{Z}_{2}$, such theories can have a $\mathbb{Z}_{2}$-valued spacetime theta angle, leading one to wonder if the two $S p(n)$ constructions give theories with different values of $\theta$, as we argued in section 5 for $S U(n)$ theories. Here is a heuristic argument that the $S p(n)$ theory made from the (B) model has $\theta=\pi$ while the other one has $\theta=0$. The (B) model has $\theta_{B}=\pi$ and fivebrane charges $(p, q)=(k-2,2 k-4)$, as we saw above. To determine the spacetime theta angle, we proceed as in section 5 . We make an $S L(2, \mathbb{Z})$ transformation

$$
\tau \rightarrow \widetilde{\tau}=\frac{\tau}{-2 \tau+1}
$$

which maps the charge vector $(k-2,2 k-4)$ to $(k-2,0)$, giving us therefore a collection of $k-2$ Dirichlet fivebranes. Reasoning as in section 5 , the spacetime theta angle is hence $\theta=2 \pi \lim _{\tau \rightarrow i \infty} \operatorname{Re} \widetilde{\tau}=\pi$. On the other hand, $S p(n)$ models derived from models of type (A) would by the same reasoning have $\theta=0$, as in this case the fivebrane charge vector is $(0,2 k-4)$ and the modular transformation is $\tau \rightarrow-1 / \tau$.

\subsection{Exceptional Groups}

It remains to briefly discuss the cases that involve exceptional gauge groups.

There are two more cases of exact sequences along the lines of (6.7). (They are Reid's examples (4) and (6).) One reads

$$
0 \rightarrow \Gamma\left(D_{4}\right) \rightarrow \Gamma\left(E_{6}\right) \rightarrow \mathbb{Z}_{3} \rightarrow 0 .
$$

The other reads

$$
0 \rightarrow \Gamma\left(E_{6}\right) \rightarrow \Gamma\left(E_{7}\right) \rightarrow \mathbb{Z}_{2} \rightarrow 0 .
$$

We have here labeled the finite subgroups of $S U(2)$ by the associated Lie groups. 
These exact sequences determine actions of $\Gamma\left(E_{6}\right)$ and $\Gamma\left(E_{7}\right)$ on $\mathbb{C}^{2} \times \mathbb{S}^{1}$ with standard action on $\mathbb{C}^{2}$ and non-trivial action on $\mathbb{S}^{1}$. (There are no analogous constructions for $E_{8}$, as $\Gamma\left(E_{8}\right)$ is simple.) With these actions, $\left(\mathbb{C}^{2} \times \mathbb{S}^{1}\right) / \Gamma\left(E_{6}\right)$ has a circle of $D_{4}$ singularities with $\mathbb{Z}_{3}$ monodromy. The monodromy acts by an outer automorphism of $D_{4}$ that breaks $D_{4}$ to $G_{2}$ (or a subgroup that can be obtained by Higgsing). So in the small radius limit, we get a six-dimensional theory with $G_{2}$ gauge group.

On the other hand, $\left(\mathbb{C}^{2} \times \mathbb{S}^{1}\right) / \Gamma\left(E_{7}\right)$ has a circle of $E_{6}$ singularities with a $\mathbb{Z}_{2}$ monodromy that breaks $E_{6}$ down to $F_{4}$ (or a subgroup that can be obtained by Higgsing). So in the small radius limit, we get a six-dimensional theory with $F_{4}$ gauge group.

To summarize what we have learned in this section, from $M$-theory on $\left(\mathbb{C}^{2} \times \mathbb{S}^{1}\right) / \Gamma$, one can obtain in a small radius limit, with a suitable action of $\Gamma$ and suitable Wilson lines, six-dimensional theories with any desired simple gauge group at low energies.

\section{Realization by Matrix Theory}

In this section, we return to the $(p, q)$ fivebrane theory and describe a matrix model realization.

First we recall from [21] (following [22, 23]) the description of matrix theory on $\mathbb{R}^{7} \times \mathbb{C}^{2} / \mathbb{Z}_{q}$. In matrix theory, one works in light cone gauge, and so in this section we will write only the transverse part of the spacetime, which in the present example is $\mathbb{R}^{5} \times \mathbb{C}^{2} / \mathbb{Z}_{q}$. To describe matrix theory with this transverse spacetime, one needs a $\mathbb{Z}_{q}$-invariant collection of zerobranes on $\mathbb{R}^{5} \times \mathbb{C}^{2}$. An important special case is that in which $\mathbb{Z}_{q}$ acts by cyclic permutations on $q$-plets of zerobranes. (Other cases correspond to a matrix description of states carrying nonzero charges under the maximal torus of the $S U(q)$ gauge symmetry of $M$-theory on $\mathbb{R}^{7} \times \mathbb{C}^{2} / \mathbb{Z}_{q}$.) In this case, the total number of zerobranes is $N q$ for some $N \geq 0$; one ultimately wishes to take $N \rightarrow \infty$.

The matrix description of this system is based on a quantum mechanical model with eight supercharges (which could come, for example, by dimensional reduction from $\mathcal{N}=2$ in four dimensions), gauge group $U(N)^{q}$, and hypermultiplets which if the $U(N)$ factors in the gauge group are correctly ordered transform as $(\mathbb{N}, \overline{\mathbb{N}}, \mathbb{1}, \ldots, \mathbb{1}) \oplus(\mathbb{1}, \mathbb{N}, \overline{\mathbb{N}}, \mathbb{1}, \ldots, \mathbb{1}) \oplus \ldots$ (a sum of $q$ terms obtained by cyclic permutations). We will recall below how this structure comes about.

Classically, this theory has several branches of vacua, parametrized by the expectation values of scalar fields $H$ in hypermultiplets and scalar fields $\Phi$ in the $U(N)^{q}$ vector multiplets. In particular, there is a branch on which the $H$ 's have generic expectation values (compatible with vanishing of the 
$D$ terms). On this branch, which we call the Higgs branch, the gauge group is broken to $U(1)^{N}$. On the Higgs branch, the expectation values of the $H$ 's parametrize the positions of $N$ zerobranes on $\mathbb{C}^{2} / \mathbb{Z}_{q}$, and the expectation values of the $\Phi$ 's parametrize the positions of the $N$ zerobranes on $\mathbb{R}^{5}$. (The unbroken $U(1)^{N}$ gauge group has a simple intuitive interpretation: there is a $U(1)$ factor for each zerobrane.) Altogether, then, this branch is related to the motion of zerobranes on the transverse space $\mathbb{R}^{5} \times \mathbb{C}^{2} / \mathbb{Z}_{q}$. There is also, classically, a Coulomb branch on which the $H$ 's are zero and the expectation values of the $\Phi$ 's break the gauge group to a maximal torus $U(1)^{N q}$; the eigenvalues of the $\Phi$ 's can be parametrized by the positions of $N q$ "particles" on $\mathbb{R}^{5}$. Quantum mechanically, wave functions from one branch leak onto another (at least for any fixed $N$ ), and there is no precise separation between them.

To consider instead $M$-theory on $\mathbb{R}^{6} \times \mathbb{C}^{2} / \mathbb{Z}_{q} \times \mathbb{S}^{1}$, that is on a transverse space $\mathbb{R}^{4} \times \mathbb{C}^{2} / \mathbb{Z}_{q} \times \mathbb{S}^{1}$, one must in the usual fashion [24] $T$-dualize in one of the coordinates. Then one gets a $1+1$-dimensional $(4,4)$ supersymmetric gauge theory with gauge group $U(N)^{q}$. In addition to the vector multiplets of $U(N)^{q}$, there are hypermultiplets transforming still as $(\mathbb{N}, \overline{\mathbb{N}}, \mathbb{1}, \ldots, \mathbb{1}) \oplus$ $(\mathbb{1}, \mathbb{N}, \overline{\mathbb{N}}, \mathbb{1}, \ldots, \mathbb{1}) \oplus \ldots$ The 1+1-dimensional theory is formulated on $\mathbb{R} \times \widetilde{\mathbb{S}}^{1}$, where $\widetilde{\mathbb{S}}^{1}$ is "dual" to the $\mathbb{S}^{1}$ in the original $M$-theory description. For reasons we have recalled in section 2 , a decoupled six-dimensional theory is expected to appear in the limit in which the radius of $\mathbb{S}^{1}$ goes to zero, and that of $\widetilde{\mathbb{S}}^{1}$ goes therefore to infinity.

Like the $0+1$-dimensional system whose behavior was summarized two paragraphs ago, this system has several branches of classical vacua. There is a Higgs branch, in which the $H$ 's have generic expectation values, and a Coulomb branch, in which the $H$ 's vanish. The former describes motion of $N$ strings on $\mathbb{R}^{4} \times \mathbb{C}^{2} / \mathbb{Z}_{q}$ and the latter describes the motion of $N q$ "strings" on a transverse $\mathbb{R}^{4}$. (These statements are closely related to the corresponding $0+1$-dimensional statements summarized above; in going from $0+1$ to $1+1$ dimensions, zerobranes become onebranes or strings and the $\Phi$ 's are reduced from describing a transverse $\mathbb{R}^{5}$ to a transverse $\mathbb{R}^{4}$.) In the limit that the radius of $\widetilde{\mathbb{S}}^{1}$ goes to infinity, the Higgs and Coulomb branches flow to separate conformal field theories, for reasons explained in [2]. Evidently, in this limit, if matrix theory is correct, the Higgs branch describes the free Type IIA theory on transverse $\mathbb{R}^{4} \times \mathbb{C}^{2} / \mathbb{Z}_{q}$, and the Coulomb branch describes the decoupled six-dimensional "gauge" theory with transverse $\mathbb{R}^{4}$.

The last statement, which has also been obtained in [6], is, roughly, "mirror" to a corresponding statement for a matrix theory realization of a six-dimensional theory related to Type IIA parallel fivebranes. In that case $[25,26]$, the conventional physics comes from a Coulomb branch and the decoupled six-dimensional physics comes from a Higgs branch. 


\section{$7.1 \quad(p, q)$ Case}

Now we come to our real interest, which is the $(p, q)$ generalization explored in sections 2-5. For this, in view of the discussion in section 3, we must consider $M$-theory on a transverse $\mathbb{R}^{4} \times\left(\mathbb{C}^{2} \times \mathbb{S}^{1}\right) / \mathbb{Z}_{q}$, where the $\mathbb{Z}_{q}$ action is as in equation 3.4 :

$$
\begin{aligned}
z_{1} & \rightarrow e^{2 \pi i / q} z_{1} \\
z_{2} & \rightarrow e^{-2 \pi i / q} z_{2} \\
t & \rightarrow t-2 \pi R \frac{p}{q}
\end{aligned}
$$

I claim that this theory has a matrix realization in terms of a $(4,4)$ supersymmetric gauge theory on $\mathbb{R} \times \widetilde{\mathbb{S}}^{1}$ which is a "twisted" version of the one just described. The gauge group is still $U(N)^{q}$, and (with a suitable cyclic ordering of the $U(N)$ factors) the hypermultiplets still transform as $(\mathbb{N}, \overline{\mathbb{N}}, \mathbb{1}, \ldots, \mathbb{1}) \oplus(\mathbb{1}, \mathbb{N}, \overline{\mathbb{N}}, \mathbb{1}, \ldots, \mathbb{1}) \oplus \ldots$ However, as one goes around the circle $\widetilde{\mathbb{S}}^{1}$, the $q$ factors in the gauge group $U(N)^{q}$ are permuted by an outer automorphism that preserves the cyclic ordering of the factors but moves each factor $p$ steps to the "left." Thus, locally on $\widetilde{\mathbb{S}}^{1}$, the theory is actually independent of $p$.

Just as in the case $p=0$, the model has a Higgs branch and a Coulomb branch at the classical level. In the limit that the radius of $\mathbb{S}^{1}$ goes to zero (and that of $\widetilde{\mathbb{S}}^{1}$ to infinity), the Higgs branch and the Coulomb branch become separated quantum mechanically. The six-dimensional model that we explored in sections 2-5 arises from the Coulomb branch.

To analyze the problem, it will be helpful to view $X_{p, q}=\left(\mathbb{C}^{2} \times \mathbb{S}^{1}\right) / \mathbb{Z}_{q}$ as $\left(\mathbb{C}^{2} \times \mathbb{R}\right) / \Gamma$, where $\Gamma$ is the discrete group generated by

$$
\begin{array}{lll}
\alpha: z_{1} \rightarrow e^{2 \pi i / q} z_{1}, & z_{2} \rightarrow e^{-2 \pi i / q} z_{2}, & t \rightarrow t-\frac{2 \pi R}{q}, \\
\beta: z_{1} \rightarrow z_{1}, & z_{2} \rightarrow z_{2}, & t \rightarrow t+2 \pi R .
\end{array}
$$

Note that $\alpha$ and $\beta$ obey the one relation

$$
\alpha^{q}=\beta^{-p} .
$$

To study $M$-theory on transverse $\mathbb{R}^{4} \times X_{p, q}$ using this description of $X_{p, q}$, we must study $\Gamma$-invariant configurations of zerobranes on $\mathbb{R}^{4} \times \mathbb{C}^{2} \times \mathbb{R}$.

Consider, in general, the action of a discrete group $\Gamma$ on a manifold $P$ and the problem of describing $\Gamma$-invariant zerobrane configurations on $P$. $\Gamma$ invariance means that the zerobrane configuration is a sum of $\Gamma$ orbits. If $\Gamma$ acts freely on $P$, each $\Gamma$ orbit is a copy of $\Gamma$ itself. In this case, the zerobrane 
positions are labeled by an element $\gamma \in \Gamma^{16}$ and by possible additional labels. If $\Gamma$ does not act freely on $P$, there are also smaller orbits, supported at fixed points of elements of $\Gamma$.

In our problem, $\Gamma$ acts freely if and only $p$ and $q$ are relatively prime. Otherwise, there are fixed points of a $\mathbb{Z}_{r}$ subgroup of $\Gamma$ ( $r$ being as before the greatest common divisor of $p$ and $q$ ) at the origin in $\mathbb{C}^{2}$. Inclusion of the non-free $\Gamma$ orbits will give, as in [21], a matrix description of states carrying non-zero charges under the maximal torus of the unbroken $S U(r)$ gauge group. For simplicity, we will here consider only the free $\Gamma$ orbits.

Just as in the case of $M$-theory on a circle, it is helpful to make $T$ duality with respect to the group $\Gamma$. This is done by introducing the unitary representations of the group $\Gamma$, a procedure that is particularly useful when $\Gamma$ is abelian, as in the present case. A unitary representation is determined by setting $\beta=e^{i \theta}$ for some real theta, and setting $\alpha$ to be a complex number (which we also call $\alpha$ ) that obeys

$$
\alpha^{q}=e^{-i p \theta} .
$$

Here $\theta$ is a coordinate on a dual circle $\widetilde{S}^{1}$ on which the matrix string theory will be formulated. Since (7.4) has $q$ solutions for each value of $\theta$, this family of $\Gamma$ representations is a $q$-fold cover $C$ of $\widetilde{\mathbb{S}}^{1}$. $C$ looks locally like $q$ copies of $\widetilde{\mathbb{S}}^{1}$, but under $\theta \rightarrow \theta+2 \pi$, the branches undergo a cyclic permutation by $p$ steps. We will find (as one would expect from [21]) that the branches correspond to the factors of $U(N)$ in the gauge group $U(N)^{q}$; the behavior under $\theta \rightarrow \theta+2 \pi$ will lead to an outer automorphism of the gauge group, cyclically permuting the factors, in going around $\widetilde{\mathbb{S}}^{1}$.

Let us consider the case that the zerobranes consist of $N$ free $\Gamma$ orbits. A component of zerobrane position can thus be labeled by $x_{i \gamma}$, with $\gamma \in \Gamma$ and $i=1, \ldots, N$. For clarity in the following, we will suppress the $i$ index, except in stating final results. Inclusion of the $i$ index has the effect of making everything $N \times N$ matrix-valued.

Let $x$ be a component of zerobrane position on which $\Gamma$ acts by $\alpha x=\zeta x$ and $\beta x=x$. Thus, in terms of the transverse space $\mathbb{R}^{4} \times\left(\mathbb{C}^{2} \times \mathbb{R}\right), x$ could be a coordinate on $\mathbb{R}^{4}$, in which case $\zeta=1$, or $x$ could be a coordinate $z_{1}$ or $z_{2}$ of $\mathbb{C}^{2}$, in which case $\zeta=e^{ \pm 2 \pi i / q}$.

With the $i$ index suppressed, $x$ is a matrix $x_{\gamma, \gamma^{\prime}}$ in a basis given by elements of $\Gamma$. If we write $\gamma=\alpha^{a} \beta^{s}$ where we can take $a=0,1, \ldots, q-1$, then $x$ is a matrix $x_{a, s ; b, t}$. To increase the legibility of the formulas, we will

\footnotetext{
${ }^{16}$ To be pedantic, a free $\Gamma$ orbit has no canonical point on it, unlike the group $\Gamma$, which has a canonical point, namely the identity element of the group. Thus the free orbit should be regarded as a "principal homogeneous space" for $\Gamma$ action, which is a copy of $\Gamma$ on which $\Gamma$ acts from (say) the left, but which is not endowed with a group structure.
} 
write this as $x(a, s ; b, t)$. The transformation law of $x$ under $\Gamma$ means that

$$
\begin{aligned}
& x(a+1, s ; b+1, t)=\zeta x(a, s ; b, t) \\
& x(a, s+1 ; b, t+1)=x(a, s ; b, t) .
\end{aligned}
$$

Now we make a Fourier transform from the basis $b, t$ to a basis of functions of $\alpha$ and $\theta$ :

$$
x\left(\alpha^{\prime}, \theta^{\prime} ; \alpha, \theta\right)=\frac{1}{2 \pi q} \sum_{b^{\prime}, t^{\prime} ; b, t} x\left(b^{\prime}, t^{\prime} ; b, t\right)\left(\alpha^{\prime}\right)^{-b^{\prime}} e^{-i t^{\prime} \theta^{\prime}} \alpha^{b} e^{i t \theta} .
$$

Using 7.5, this becomes

$$
\begin{aligned}
& x\left(\alpha^{\prime}, \theta^{\prime} ; \alpha, \theta\right)=\frac{1}{2 \pi q} \sum_{b^{\prime}, t^{\prime} ; b, t} x\left(b^{\prime}-b, t^{\prime}-t ; 0,0\right) \\
& \cdot\left(\zeta \alpha\left(\alpha^{\prime}\right)^{-1}\right)^{b}\left(\alpha^{\prime}\right)^{b-b^{\prime}} e^{i t\left(\theta-\theta^{\prime}\right)} e^{i\left(t-t^{\prime}\right) \theta^{\prime}} .
\end{aligned}
$$

The sum over $b$ and $t$ for fixed $b-b^{\prime}, t-t^{\prime}$ now gives delta functions setting

$$
\begin{aligned}
\theta^{\prime} & =\theta \\
\alpha^{\prime} & =\zeta \alpha .
\end{aligned}
$$

The first equation says that $x$ acts locally in $\theta$. Reintroducing the so far suppressed index $i=1, \ldots, N, x$ acts at given $\theta$ on a space of dimension $N q$; a basis of this space is labeled by $i$ and by the choice of $\alpha$ with $\alpha^{q}=e^{-i p \theta}$. However, the second equation in (7.8) says that for $\zeta=1, x$ acts diagonally on the $\alpha$ index. Thus, the $x$ 's that have $\zeta=1$ split up at fixed $\theta$ as the sum of $q$ different $N \times N$ blocks, one for each value of $\alpha$.

These $x$ 's transform in the adjoint representation of $U(N)^{q}$, and are in fact the scalar fields in the vector multiplet.

On the other hand, if $\zeta=e^{ \pm 2 \pi i / q}$, then the second equation in (7.8) says that multiplication by $x$ makes a cyclic permutation on the set of possible values of $\alpha$ by \pm 1 step. As a result, these components of $x$, which are the coordinates $z_{1}, z_{2}$ of $\mathbb{C}^{2}$, transform under the gauge group as $(\mathbb{N}, \overline{\mathbb{N}}, \mathbb{1}, \ldots, \mathbb{1}) \oplus$ $\ldots$ in the case of $z_{1}$, or $(\overline{\mathbb{N}}, \mathbb{N}, \mathbb{1}, \ldots, \mathbb{1}) \oplus \ldots$ for $z_{2}$. (In each case, .. refers to terms obtained via cyclic permuations.) These fields are the bosonic part of $1+1$-dimensional hypermultiplets transforming as $(\mathbb{N}, \overline{\mathbb{N}}, \mathbb{1}, \ldots, \mathbb{1}) \oplus$ cyclic permutations.

It remains to make a similar analysis for the remaining coordinate $t$, which according to 7.1 transforms inhomogeneously under $\Gamma$. In this case, 
we have

$$
\begin{aligned}
t\left(\alpha^{\prime}, \theta^{\prime} ; \alpha, \theta\right)= & \frac{1}{2 \pi q} \sum_{b^{\prime}, u^{\prime} ; b, u} t\left(b^{\prime}, u^{\prime} ; b, u\right)\left(\alpha^{\prime}\right)^{-b^{\prime}} e^{-i u^{\prime} \theta^{\prime}} \alpha^{b} e^{i u \theta} \\
= & \frac{1}{2 \pi q} \sum_{b^{\prime}, u^{\prime} ; b, u}\left(t\left(b^{\prime}-b, u^{\prime}-u ; 0,0\right)+2 \pi R\left(u-\frac{p}{q} b\right)\right) \\
& \cdot\left(\alpha^{\prime}\right)^{-\left(b^{\prime}-b\right)} e^{-i\left(u^{\prime}-u\right) \theta^{\prime}}\left(\alpha\left(\alpha^{\prime}\right)^{-1}\right)^{b} e^{i\left(u-u^{\prime}\right) \theta} .
\end{aligned}
$$

The term on the right which is homogeneous in $t$ gives, after summing over $b$ and $u$ for fixed $b^{\prime}-b$ and $u^{\prime}-u$, a contribution proportional to $\delta\left(\theta^{\prime}-\theta\right) \delta_{\alpha^{\prime}, \alpha}$. This contribution is local in $\theta$ and diagonal in $\alpha$ and corresponds, after remembering to include the additional label $i=1, \ldots, N$, to a function $A(\theta)$ with values in the adjoint representation of $U(N)^{q}$. This will be interpreted as the gauge field on $\widetilde{\mathbb{S}}^{1}$.

The $2 \pi R u$ term on the right hand side gives after the sum over $u$ a term $-i \delta^{\prime}\left(\theta-\theta^{\prime}\right)$, which is the matrix element of $-i d / d \theta$. Adding this to the term analyzed in the last paragraph would give a covariant derivative $-i D / D \theta=-d / d \theta+A$. At first sight, the $2 \pi R b(-p / q)$ term on the right of (7.9) is obscure. However, we must remember that because of the relation $\alpha^{q}=e^{-i p \theta}$, we cannot vary $\theta$ keeping $\alpha$ fixed. If we change variables from $\theta$ and $\alpha$ to $\theta$ and $\widetilde{\alpha}=\alpha e^{i(p / q) \theta}$ (which can remain fixed as $\theta$ varies) then in the $e^{i u\left(\theta-\theta^{\prime}\right)}$ factor in $(7.9), u$ is replaced by $u-(p / q) b$. So the inhomogeneous term on the right hand side of (7.9) is the Fourier transform of $-i d /\left.d \theta\right|_{\widetilde{\alpha}}$, the $\theta$ derivative at fixed $\widetilde{\alpha}$. The net effect is that $t$ is interpreted as a covariant derivative at fixed $\widetilde{\alpha}$,

$$
t \rightarrow-\left.i \frac{D}{D \theta}\right|_{\widetilde{\alpha}}
$$

Varying $\theta$ at fixed $\widetilde{\alpha}$ means that under $\theta \rightarrow \theta+2 \pi, \alpha$ is transformed to $\alpha e^{-2 \pi i(p / q)}$, as we have claimed. This transformation of $\alpha$ in going around $\widetilde{\mathbb{S}}^{1}$ is the one essentially new point in our analysis, compared to the familiar case of $p=0$. Since the $U(N)$ factors in the gauge group are associated with the possible values of $\alpha$, this shift means that in going around $\widetilde{\mathbb{S}}^{1}$, the gauge group $U(N)^{q}$ is transformed by an outer automorphism that moves each factor $p$ steps to the left.

\section{Acknowledgments}

I would like to thank S. Shenker and H. Verlinde for raising questions that stimulated this study, D. Morrison for comments and for pointing out reference [20], H. Ooguri and S. Sethi for discussions, A. Karch and B. Kol for pointing out an error in an earlier version of section 5, and the Aspen Center for Physics for its hospitality. 


\section{References}

[1] N. Seiberg, Phys. Lett. B408 (1997) 98.

[2] E. Witten, "Some Comments On String Dynamics", hep-th/9507121.

[3] A. Strominger, Phys. Lett. B383 (1996) 44.

[4] J. Blum and K. Intriligator, Nucl. Phys. B506 (1997) 199.

[5] C. Vafa, Adv. Theor. Math. Phys. 1 (1997) 158.

[6] S. Sethi, "The Matrix Formulation of Type IIB Five-Branes", hepth/9710005.

[7] H. Ooguri and C. Vafa, Nucl. Phys. B463 (1996) 55.

[8] M. Bershadsky, V. Sadov, and C. Vafa, Nucl. Phys. B463 (1996) 398.

[9] A. Sen, "A Note on Enhanced Gauge Symmetries In M-And String Theory", hep-th/9707123.

[10] R. Gregory, J. A. Harvey, G. Moore, Adv. Theor. Math. Phys. 1 (1997) 283.

[11] J. Schwarz and A. Sen, Phys. Lett. B357 (1995) 323.

[12] P. Aspinwall and M. Gross, Phys. Lett. B387 (1996) 735.

[13] M. Bershadsky, K. Intriligator, S. Kachru, D.R. Morrison, V. Sadov, and C. Vafa, Nucl. Phys. B481 (1996) 215.

[14] C. Hull and P. Townsend, Nucl. Phys. B438 (1995) 109.

[15] N. Seiberg and S. Sethi, Adv. Theor. Math. Phys. 1 (1997) 259.

[16] M. Green, J. Harvey, and G. Moore, "I-Brane Inflow And Anomalous Couplings On D-Branes", Class. Quant. Grav. 14 (1997) 47.

[17] B. Kol, “On 6-D 'Gauge' Theories With Irrational Theta Angle”, hepth/9711017.

[18] E. Witten, Nucl. Phys. B443 (1995) 85.

[19] P. S. Aspinwall, S. Katz, and D. R. Morrison, to appear.

[20] M. Reid, "Young Person's Guide To Canonical Singularities" in "Algebraic Geometry", Proc. Symp. Pure Math. 46 (1985) 345. 
[21] M. Douglas, "Enhanced Gauge Symmetry In M(atrix) Theory", hepth/9612126.

[22] M. Douglas and G. Moore, "D-Branes, Quivers, and ALE Instantons", hep-th/9603167.

[23] C. V. Johnson and R. C. Myers, Nucl. Phys. B484 (1997) 653.

[24] W. Taylor, Phys. Lett. B394 (1997) 283.

[25] O. Aharony, M. Berkooz, S. Kachru, N. Seiberg, E. Silverstein, Adv. Theor. Math. Phys. 1 (1997) 148.

[26] E. Witten, "On The Conformal Field Theory Of The Higgs Branch", hep-th/9707093. 